\title{
Enhanced Surveillance of Heat-Related Illness in Pinal County
}

\author{
Dylan C. Kent ${ }^{\star 1}$, Rachel Z. Garcia ${ }^{2}$, Samuel Packard², Graham Briggs², Clancey Hill², \\ Stephanie Griffin 1 , Edward Bedrick ${ }^{1}$, Krystal Collier ${ }^{3}$ and Matthew Roach ${ }^{3}$
}

${ }^{1}$ Public Health, University of Arizona, Tucson, AZ, USA; ${ }^{2}$ Pinal County Public Health Services District, Florence, AZ, USA; ${ }^{3}$ Arizona

Department of Health Services, Phoenix, AZ, USA

\section{Objective}

Using a syndromic surveillance system to understand the magnitude and risk factors related to heat-related illness (HRI) in Pinal County, AZ.

\section{Introduction}

Extreme heat is a major cause of weather-related morbidity and mortality in the United States (US). ${ }^{1}$ HRI is the most frequent cause of environmental exposure-related injury treated in US emergency departments. ${ }^{2}$ More than 65,000 emergency room visits occur for acute HRI each summer nationwide. ${ }^{3}$ In Arizona, HRI accounts for an estimated 2,000 emergency room patients and 118 deaths each year. ${ }^{4}$ As heat-related illness becomes increasingly recognized as a public health issue, local health departments are tasked with building capacity to conduct enhanced surveillance of HRI in order to inform public health preparedness and response efforts. In Pinal County, understanding the magnitude and risk factors of HRI is important for informing prevention efforts as well as developing strategies to respond to extreme heat.

\section{Methods}

To gain a better understanding of the magnitude of HRI in Pinal County, historical cases were reviewed from hospital discharge data (HDD) from 2010-2016. Cases were included if the discharge record included any ICD codes consistent with HRI (ICD-9 codes 992 or ICD-10 codes T67 or X30) and if the patient's county of residence was Pinal County. Recent HRI cases during the summer of 2017 were identified using the National Syndromic Surveillance Program BioSense Platform. The ESSENCE syndromic surveillance tool within the BioSense Platform includes data reported by local hospitals. This data can be used to detect abnormal activity for public health investigation. HRI cases were identified in ESSENCE based on ICD-10 codes and chief complaint terms according to a standardized algorithm developed by the Council of State and Territorial Epidemiologists. ${ }^{1}$ Both emergency department and admitted patients with a HRI were abstracted from HDD and ESSENCE. To assess HRI risk factors for the summer of 2017, a survey instrument was developed. Survey questions included the nature and location of the HRI incident, potential risk factors, and knowledge and awareness of HRI. Cases were identified in ESSENSE on a weekly basis from May 1, 2017-September 12, 2017, and follow up phone interviews were conducted with eligible cases. For HRI cases eligible for interview, three attempts were made to contact the patient by phone. Cases were excluded if the patient was incarcerated, deceased, or did not have a HRI upon medical record review. An exploratory analysis was performed for the data from HDD, ESSENCE, and interviews.

\section{Results}

Pinal County Public Health Services District identified 1,321 HRI cases from 2010-2016, an average of 189 per year. Hospital discharge data suggest HRI cases are more likely to occur in males between the ages of $20-44$ years old (27\%). It is also notable that a sharp increase in HRI cases is observed each year in mid-to-late June, with an estimated $14 \%$ of annual cases occurring during the third week of June. Further analysis of HDD showed 31\% of cases received medical treatment in Casa Grande in central Pinal County. Between May 1st and September 12th of 2017, 161 HRI cases were detected using ESSENCE. Of which 149 cases were determined to be HRI; 22 cases did not have contact information, and 4 cases were ineligible due to incarceration or death. A total of 31 HRI cases were interviewed out of the eligible 123 ESSENSE cases (25\% response rate). Interview data indicated occupational exposure to extreme heat as a major risk factor for HRI. Additional risk factors reported during interviews included exposure to extreme heat while at home or traveling, although interview results are not representative due to a small sample size $(\mathrm{n}=31)$.

\section{Conclusions}

Syndromic surveillance combined with interviews and a review of HDD provides an informative approach for monitoring and responding to HRI. Data suggest Pinal County should expect an increase in HRI cases by mid-June each year, typically coinciding with the first National Weather Service Extreme Heat Warning of the season. Preliminary results suggest that cases occur more frequently in working males ages 20-44 years old in occupations that expose workers to extreme heat conditions. Additional information is needed to assess risk factors for HRI among vulnerable populations in Pinal County who were not represented in this study, including individuals who are homeless, undocumented, elderly, or in correctional facilities. Future areas for improvement include improving the phone interview script to include English and Spanish language versions and performing medical record abstractions on all HRI cases. Enhanced syndromic surveillance is recommended to provide information on risk factors for HRI to inform prevention efforts in Pinal County.

\section{Keywords}

heat-related illness; hospital discharge data; ESSENCE

\section{Acknowledgments}

Thank you to our colleagues at the Arizona Department of Health Services, Arizona State Univeristy, and the University of Arizona.

\section{References}

1. Heat-Related Illness Syndrome Query: A Guidance Document For Implementing Heat-Related Illness Syndromic Surveillance in Public Health Practice. In: Epidemiologists CoSaT, ed. Vol 1.02016:1-12.

2. Pillai SK, Noe RS, Murphy MW, et al. Heat illness: predictors of hospital admissions among emergency department visits-Georgia, 2002-2008. J Community Health. 2014;39(1):90-98.

3. Centers for Disease Control and Prevention. Climate Change and Extreme Heat: What You Can Do to Prepare. 2016; Available from https:// www.cdc.gov/climateandhealth/pubs/extreme-heat-guidebook.pdf

4. Trends in Morbidity and Mortality from Exposure to Excessive Natural Heat in Arizona, 2012 report. In: Services ADoH, ed2012.

\section{*Dylan C. Kent}

E-mail: dkent@email.arizona.edu 